\title{
Rituximab as a monotherapy or in combination therapy for the treatment of non-paraneoplastic autoimmune retinopathy
}

This article was published in the following Dove Press journal:

Clinical Ophthalmology

17 February 2017

Number of times this article has been viewed

\author{
Arash Maleki ${ }^{1,2}$ \\ Neerav Lamba ${ }^{1,2}$ \\ Lina $M a^{1,2}$ \\ Stacey Lee ${ }^{1,2}$ \\ Alexander Schmidt ${ }^{1,2}$ \\ C Stephen Foster ${ }^{1-3}$ \\ 'Massachusetts Eye Research \\ and Surgery Institution, ${ }^{2}$ Ocular \\ Immunology and Uveitis Foundation, \\ Waltham, ${ }^{3}$ Harvard Medical School, \\ Boston, MA, USA
}

Purpose: To examine the efficacy of rituximab as a monotherapy or in combination therapy for the treatment of patients with non-paraneoplastic autoimmune retinopathy.

Methods: Twelve eyes of six patients with non-paraneoplastic autoimmune retinopathy who were treated with rituximab and had at least 6 months of follow-up were included. Demographic data, clinical data, visual field parameters, electroretinography parameters, and anti-retinal and anti-optic nerve autoantibody bands were collected from the Massachusetts Eye Research and Surgery Institution database between September 2010 and January 2015. Changes in visual acuity, visual field parameters, electroretinography parameters, and anti-retinal and anti-optic nerve autoantibody bands from the initial visit to the most recent visit were examined.

Results: From the initial visit to the last visit, visual acuity was stable in eight (66.7\%) eyes. Visual field was stable in six (50\%) eyes and improved in two (16.7\%) eyes. Electroretinography was stable or improved in eight $(66.7 \%)$ eyes. The average number of anti-retinal and anti-optic nerve antibody bands was reduced.

Conclusion: Stabilization and/or improvement of visual acuity, visual field parameters, and electroretinography parameters were observed in a high number of patients (75\%) on rituximab, as a monotherapy (one patient) or in combination therapy.

Keywords: birdshot retinochoroidopathy, bortezomib, CD20, cyclophosphamide, HLA-A29, HLA-B27, immunomodulatory therapy, systemic lupus erythematosus

\section{Introduction}

Autoimmune retinopathy (AIR) is an immune-mediated retinopathy that can cause acute, subacute and chronic vision loss, visual field defects, photopsias, nyctalopia, and color vision defects. ${ }^{1,2}$ AIR is characterized by the presence of circulating anti-retinal antibodies that are believed to damage photoreceptors in the retina; however, the exact mechanism is not entirely understood. . $^{3,4}$

AIR is subdivided into paraneoplastic (cancer-associated retinopathy and melanomaassociated retinopathy) and non-paraneoplastic categories. Non-paraneoplastic AIR is composed of a large group of autoimmune retinopathies with features similar to those of carcinoma-associated retinopathy, but without any known underlying malignancy. Non-paraneoplastic AIR is usually diagnosed at a younger age, ${ }^{4}$ in people with a known personal and/or familial history of autoimmune diseases., , $^{2,6}$

Long-term immunosuppressive therapy is considered the mainstay of treatment for AIR. ${ }^{7}$ Different regimens of immunomodulatory therapy, such as corticosteroid, ${ }^{8}$ plasmaphoresis, ${ }^{9}$ intravenous immunoglobulin, ${ }^{10}$ cyclosporine,${ }^{6}$ and mycophenolate mofetil, ${ }^{6}$ have been used in the treatment of paraneoplastic and non-paraneoplastic AIR.
Correspondence: C Stephen Foster Massachusetts Eye Research and Surgery Institution, 440 Main Street, Suite 20I,

Waltham, MA 0245I, USA

Tel + I 78| 89| 6377

Fax $+\mid$ 78| 647 |430

Email sfoster@mersi.com $\mathrm{BY}$
hC for commercial use of this work, please see paragraphs 4.2 and 5 of our Terms (https://www.dovepress.com/terms.php). 
However, the rarity of this entity combined with difficulty in confirming the diagnosis and monitoring the response to treatment makes treatment challenging. ${ }^{5}$ Rituximab, a cluster of differentiation 20 molecule inhibitor (CD20), has been used as a treatment option for carcinoma-associated retinopathy in limited studies. ${ }^{11,12}$ There is also a case report using rituximab in the treatment of non-paraneoplastic AIR. ${ }^{13}$ The aim of this study is to examine the efficacy of rituximab as a monotherapy or in combination therapy in preserving or improving visual function parameters in patients with non-paraneoplastic AIR.

\section{Methods}

This was a single-center, retrospective interventional case series of patients with non-paraneoplastic AIR who were treated at the Massachusetts Eye Research and Surgery Institution (MERSI) between September 2010 and January 2015. Each patient had at least 6 months of follow-up from when the diagnosis of non-paraneoplastic AIR was made. Ethical approval for this study was obtained through the New England Institutional Review Board, which issued a waiver of written informed consent based on standard operating procedures for retrospective chart reviews. This study was performed in accordance with the Declaration of Helsinki and was Health Insurance Portability and Accountability Act compliant.

Diagnosis of AIR was made based on four essential criteria with subjective symptoms as supportive criteria. ${ }^{5}$ Essential criteria included no evidence of systemic malignancies or infections after a complete history was taken and a physical examination and appropriate testing were performed; no evidence of a preexisting degenerative eye disease; confirmed progressive abnormality on repeated electroretinography and/or visual field; and the presence of anti-retinal and/or anti-optic nerve antibodies. Supportive criteria were the acute or subacute presence of symptoms, such as decreased vision, visual field defects, nyctalopia, photoaversion, photopsia, and color vision deficits in patients without active anterior or posterior uveitis.

Blood samples and pertinent medical history of each patient were collected at MERSI and were sent to the commercially available testing center at Oregon Health Sciences University. Western blots were used to evaluate the presence of the anti-retinal and anti-optic nerve antibodies. These tests were repeated every 6 months.

All patients underwent fluorescein angiography, indocyanine green (ICG) angiography, full-field electroretinography, and automated perimetry. Optical coherence tomography was obtained as part of the initial examination or follow-up examinations in all patients with clinical evidence of decreased central visual acuity at any time or angiographic suspicion of macula edema.

Patient demographics, including personal and family histories of autoimmune diseases, were collected. Best corrected visual acuity and related logarithm of the minimum angle of resolution (LogMAR) were recorded for every patient at the initial and most recent visits. Humphrey visual field (HVF; Carl Zeiss Meditec, Inc., Dublin, CA, USA) and Ganzfeld electroretinography (SG-2002, LKC Technologies, Gaithersburg, MD) were also performed on all patients at the initial and most recent visits. Anti-retinal and anti-optic nerve autoantibody bands, reported based on their molecular weights $(\mathrm{kDa})$, were recorded at the initial and most recent visits. Furthermore, anti-retinal and anti-optic nerve antibodies were divided into pathologically proven and nonspecific groups, as based on the previous studies. ${ }^{2,5,14}$ After a thorough analysis of the patient's records, treatment regimens, the duration of each regimen, reported clinical and laboratory (blood and urine) side effects, and reasons for premature termination of a treatment regimen and its related date were recorded for every patient in the study.

Visual fields were analyzed with the Humphrey Visual Field Analyzer (Carl Zeiss Meditec, Inc.) under 24-2 FASTPAC or Swedish Interactive Threshold Activity (SITA) fast strategy for all patients, except those with a previous diagnosis of birdshot retinochoroidopathy. Short-wavelength automated perimetry (SITA-SWAP) was used to evaluate the visual fields of patients with a previous diagnosis of birdshot retinochoroidopathy as it has been shown to be more accurate in these patients. ${ }^{15}$ Visual field parameters recorded were total deviation, pattern deviation, mean deviation, and pattern standard deviation. The standardized visual field criteria of the Optic Neuritis Treatment Trial protocol were used to interpret visual field patterns. ${ }^{16,17} \mathrm{~A}$ field defect was defined as three or more significant $(P<0.05)$ non-edge contiguous points, with at least one at the $(P<0.01)$ level on the same side of the horizontal meridian. ${ }^{18,19}$ Ganzfeld electroretinography (SG-2002, LKC Technologies) parameters included scotopic b-wave amplitude, maximal combined a-wave and b-wave amplitudes, 30-Hz flicker amplitude, and $30-\mathrm{Hz}$ flicker implicit time. The percentage of each of these parameters to the mean normal control values were calculated for statistical analysis.

The treatment protocol for rituximab was $375 \mathrm{mg} / \mathrm{m}^{2}$ every week for 8 weeks, then $375 \mathrm{mg} / \mathrm{m}^{2}$ monthly. ${ }^{20,21}$ Oral cyclophosphamide was used at a dose of $2 \mathrm{mg} / \mathrm{kg} / \mathrm{day}$. Bortezomib was the other medication, used at a dose of 
$1.3 \mathrm{mg} / \mathrm{m}^{2}$ through the subcutaneous route at days $1,4,8$, and 11; this was repeated every 21 days. All patients were monitored closely for occult side effects of all medications via a complete review of systems at each visit and periodical blood tests, including blood cell counts, blood urea nitrogen, creatinine, and liver function tests. In patients on cyclophosphamide, urine samples were analyzed for hematuria every other month. The decision for rituximab monotherapy or in combination therapy was made based on the severity of the disease.

Severe disease was defined as visual acuity 20/200 or worse; or mean deviation worse than $-12 \mathrm{~dB},>50 \%$ of points depressed $<5 \%$ level and $>25 \%$ depressed $<1 \%$ level on pattern deviation plot, any point within $5^{\circ}$ of fixation $0 \mathrm{~dB}$. Both hemifield contain point(s) $<15 \mathrm{~dB}$ within $5^{\circ}$ of fixation; ${ }^{22}$ or flat or near flat $30-\mathrm{Hz}$ flicker amplitude and/or combined a-wave and b-wave amplitudes.

We looked for stability or improvement of visual acuity, HVF patterns and parameters (total deviation, pattern deviation, mean deviation, and pattern standard deviation), and electroretinography parameters (scotopic rod response amplitude, maximal combined a-wave and b-wave amplitudes, 30-Hz flicker amplitude, and 30-Hz flicker implicit time) at the most recent visit. Stability in visual acuity was defined as a visual acuity that was similar to the first visit (within one line on the Snellen chart). Improvement in visual acuity was defined as visual acuity that improved by at least two lines on the Snellen chart from the first visit. Stability of visual field was defined as the patient having the mean deviation and pattern standard deviation within the same category based on probability symbols on HVF printout. Visual field patterns were considered improved if scotomas in the field became more localized or less dense in the final visit than in the initial visit. Finally, electroretinography parameters were considered stable when they were within $25 \%$ (minimum reported for stability) of the baseline values or better, ${ }^{23}$ respectively, from the first to the last visit.

As there were multiple parameters in the HVF and electroretinography tests, stability or improvement in each test was defined as stability or improvement in $>50 \%$ of the parameters of that test. Parameters included total deviation, pattern deviation, mean deviation, and pattern standard deviation in HVF; and scotopic rod response amplitude, maximal combined a-wave and b-wave amplitudes, $30-\mathrm{Hz}$ flicker amplitude, and $30-\mathrm{Hz}$ flicker implicit time in electroretinography. The test was determined to be inconclusive when $50 \%$ of the parameters were worse and the other $50 \%$ were stable or better.

\section{Statistical analysis}

Statistical Package for the Social Sciences (SPSS) 16.0 (IBM Corp., Armonk, NY, USA) and MedCalc software 8.2.0.3 (MedCalc ${ }^{\circledR}$, Mariakerke, Belgium) were used for statistical analysis.

\section{Results}

There were six patients (12 eyes) who met all inclusion criteria. The median age at initial presentation was 49 years (range, 30-73). The median duration between onset of symptoms and initial consultation was 10.5 months (range, 2-44). The median duration of follow-up was 18 months (range, 8-49). Of the six patients, five $(83.3 \%)$ were females. All patients were of a non-Hispanic Caucasian ethnicity. One patient had a previous diagnosis of systemic lupus erythematosus (patient 3), two patients had a previous diagnosis of birdshot retinochoroidopathy (patients 5 and 6), and one patient had a previous diagnosis of HLA-B27-positive panuveitis and vasculitis (patient 2). All patients' family histories were negative for any systemic autoimmune, ocular autoimmune, and hereditary degenerative diseases.

At the initial visit, the mean LogMAR visual acuity was $0.58 \pm 0.6(20 / 70-20 / 80)$. The presenting symptoms included progressive decreased vision in four eyes of two patients, progressive decreased vision and visual field defects in four eyes of two patients, and only progressive visual field defects in two eyes of one patient. Two eyes of one patient with birdshot retinochoroidopathy were found, incidentally, during regular monitoring with impressive changes in his visual field and electroretinography tests after almost 4 years of being in remission off medications and without any intraocular inflammation. None of the eyes had active uveitis at the initial visit. Two of the six patients (four eyes) had abnormal funduscopy examinations; one of them (two eyes) showed disc pallor, arterial narrowing, and diffuse retinal and retinal pigment epithelium atrophy, whereas the other patient (two eyes) showed isolated generalized retinal and retinal pigment epithelium atrophy. All other patients had normal funduscopy examinations bilaterally.

Fluorescein angiography did not reveal any abnormalities in any of the 12 eyes at the initial visit. ICG angiography was normal in eight eyes of four patients and was considered stable in patients who had previous diagnoses of birdshot retinochoroidopathy. Macular optical coherence tomography was obtained in four patients due to decreased visual acuity. Results of this testing showed decreased macular thickness with no macular edema or cystic changes in the macula in eight eyes of these four patients. 
Table I Non-paraneoplastic autoimmune retinopathy treatment regimens

\begin{tabular}{lllll}
\hline Patient no/sex/age & $\begin{array}{l}\text { Treatment } \\
\text { regimen }\end{array}$ & $\begin{array}{l}\text { Duration of } \\
\text { treatment (months) }\end{array}$ & $\begin{array}{l}\text { Reason for treatment } \\
\text { termination }\end{array}$ & $\begin{array}{l}\text { Total duration of } \\
\text { follow-up (months) }\end{array}$ \\
\hline I/F/30 & $R+C+B$ & 10 & Side effect to $R$, C, and B & 17 \\
$2 / F / 47$ & $R+C$ & 18 & Continued to the last visit & 18 \\
$3 / F / 5 I$ & $R$ & Continued to the last visit & 20 \\
$4 / F / 73$ & $R$ & 10 & Insurance issue & 49 \\
$5 / M / 54$ & $R+C$ & 18 & Side effect to C & 18 \\
$6 / F / 40$ & $R+B$ & 8 & Continued to the last visit & 8 \\
\hline
\end{tabular}

Abbreviations: B, bortezomib; C, cyclophosphamide; F, female; M, male; R, rituximab.

All patients received immunomodulatory therapy. Four patients with underlying systemic or ocular autoimmune diseases were already on conventional immunomodulatory therapy, which included methotrexate, cyclosporine, mycophenolate mofetil, and adalimumab. All of these medications were stopped before using the AIR treatment protocol.

Rituximab was started in all the patients in the study. Oral cyclophosphamide was used in three patients. One patient received bortezomib in combination with rituximab as the initial therapy. Cyclophosphamide was replaced with bortezomib in one patient because of documented leukopenia.

Treatment was prematurely stopped in two patients. One patient discontinued treatment due to intolerable side effects of the medications: rituximab, cyclophosphamide, and bortezomib. This patient experienced dermatitis (1 month after starting treatment), leukopenia (6 months after starting treatment), and peripheral neuropathy (2 months after starting treatment) with rituximab, cyclophosphamide, and bortezomib, respectively. The other patient discontinued treatment due to issues in insurance coverage ( 5 months after starting treatment). One patient, who was on rituximab and cyclophosphamide combination therapy, had to discontinue cyclophosphamide due to leukopenia (10 months after treatment), but was able to continue treatment with rituximab. The mean number of medications used to treat was 1.83 (range, 1-3). The average duration of treatment was 14.1 \pm 5.07 months (range, 8-20). Table 1 shows the details of the treatment regimens in each patient.

Changes in vision, visual field patterns and parameters, and electroretinography parameters from the initial visit to the most recent visit are shown in Table 2 and Figure 1.

From the initial visit to the last visit recorded, visual acuity was found to be stable in eight $(66.7 \%)$ eyes and decreased in four $(33.3 \%)$ eyes. Visual field as a single test showed stability in six (50\%) eyes, improvement in two (16.7\%) eyes, and worsening in one $(8.33 \%)$ eye. It was inconclusive in three eyes (25\%). Figure 2 shows the improvement of mean deviation and stability of pattern standard deviation in one of the patients who responded to rituximab. Electroretinography as a single test showed improvement in two (16.7\%) eyes, worsening in four $(33.3 \%)$ eyes, and stability in two $(16.7 \%)$ eyes. In four (33.3\%) eyes, electroretinography

Table 2 Visual acuity, visual field parameters, and electroretinography parameters changes from the initial visit to the most recent visit in patients with non-paraneoplastic autoimmune retinopathy

\begin{tabular}{|c|c|c|c|c|c|c|c|c|c|c|c|}
\hline $\begin{array}{l}\text { Patient } \\
\text { no/sex/ } \\
\text { age/eye }\end{array}$ & $\begin{array}{l}\text { Change }^{a} \\
\text { in VIA }\end{array}$ & $\begin{array}{l}\text { Change }^{\mathrm{a}} \\
\text { in HVF, } \\
\text { MD }\end{array}$ & $\begin{array}{l}\text { Change }^{a} \\
\text { in HVF, } \\
\text { PSD }\end{array}$ & $\begin{array}{l}\text { Change }^{a} \\
\text { in HVF, } \\
\text { TD }\end{array}$ & $\begin{array}{l}\text { Change }^{a} \\
\text { in HVF, } \\
\text { PD }\end{array}$ & $\begin{array}{l}\text { Change }^{a} \\
\text { in ERG, } \\
\text { Sc b-wave }\end{array}$ & $\begin{array}{l}\text { Change }^{a} \\
\text { in ERG, } \\
\text { C a-wave }\end{array}$ & $\begin{array}{l}\text { Change }^{a} \\
\text { in ERG, } \\
\text { C b-wave }\end{array}$ & $\begin{array}{l}\text { Change }^{\mathrm{a}} \\
\text { in ERG, } \\
\text { 30-Hz Am }\end{array}$ & $\begin{array}{l}\text { Change }^{a} \\
\text { in ERG, } \\
30-\mathrm{Hz} \text { Im }\end{array}$ & $\begin{array}{l}\text { Negative } \\
\text { wave- } \\
\text { forms }\end{array}$ \\
\hline I/F/30/OD & W & $S$ & $S$ & I & $S$ & W & W & I & W & $S$ & No \\
\hline I/F/30/OS & W & S & $S$ & $S$ & $S$ & w & w & w & w & w & No \\
\hline 2/F/47/OD & $S$ & $S$ & $S$ & $S$ & $S$ & w & $S$ & I & I & $S$ & Yes \\
\hline 2/F/47/OS & W & $S$ & $S$ & $S$ & 1 & I & W & I & I & W & Yes \\
\hline 3/F/5I/OD & $S$ & W & $S$ & W & W & $S$ & W & $S$ & 1 & 1 & No \\
\hline 3/F/5I/OS & $\mathrm{S}$ & $S$ & $S$ & w & W & I & $S$ & $S$ & w & $S$ & Yes \\
\hline 4/F/73/OD & W & $S$ & $S$ & $S$ & $S$ & w & w & w & $S$ & $S$ & No \\
\hline 4/F/73/OS & $S$ & $S$ & $S$ & $S$ & $S$ & w & W & W & W & I & No \\
\hline 5/M/54/OD & $S$ & I & $S$ & I & I & I & $S$ & $S$ & W & I & No \\
\hline 5/M/54/OS & $S$ & $S$ & W & $S$ & W & 1 & I & $S$ & W & I & No \\
\hline 6/F/40/OD & $S$ & S & $S$ & w & w & $S$ & $S$ & $S$ & 1 & I & No \\
\hline 6/F/40/OS & $S$ & I & I & 1 & 1 & $S$ & W & W & I & I & No \\
\hline
\end{tabular}

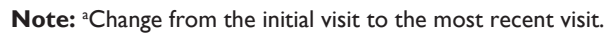

Abbreviations: Am, amplitude; C, combined; ERG, electroretinography; F, female; HVF, Humphrey visual field; I, improved; Im, implicit time; M, male; MD, mean deviation; OD, right eye; OS, left eye; PD, pattern deviation; PSD, pattern standard deviation; S, stable; Sc, Scotopic; TD, total deviation; V/A, visual acuity; W, worse. 
A

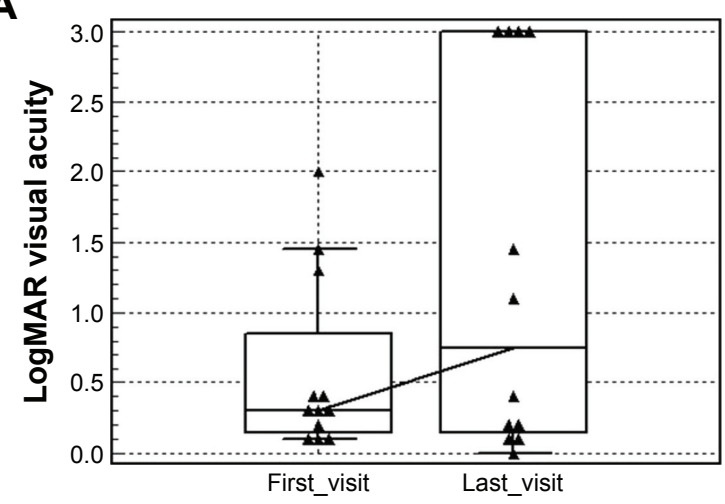

C

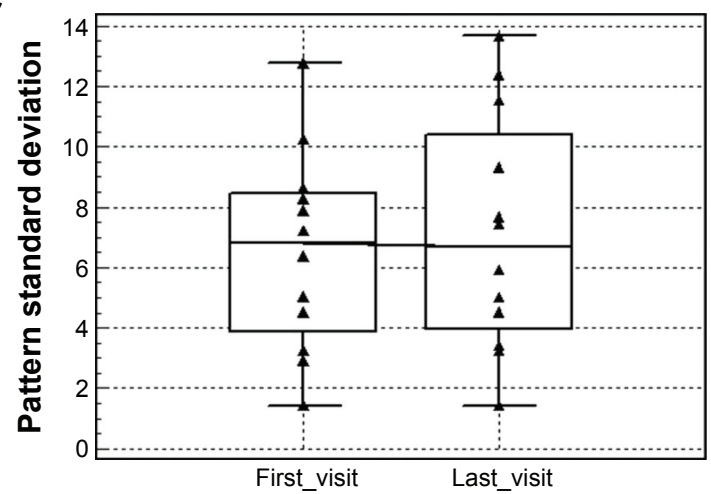

E

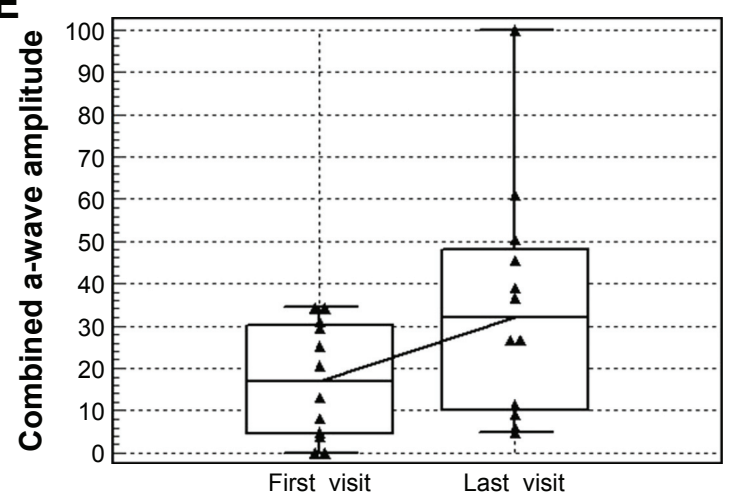

G

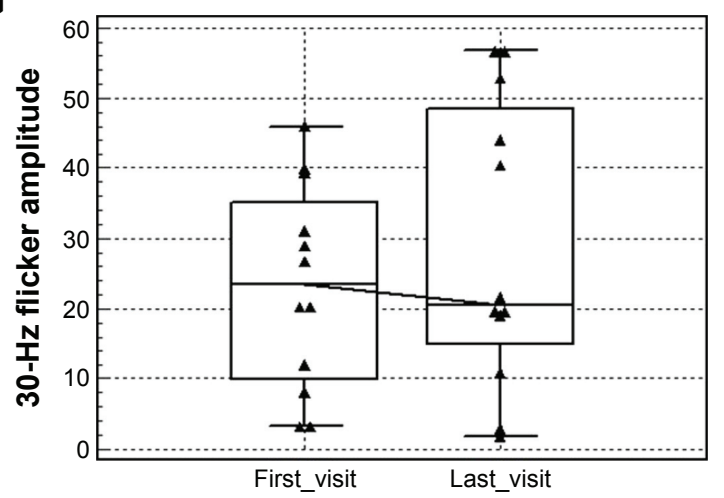

B

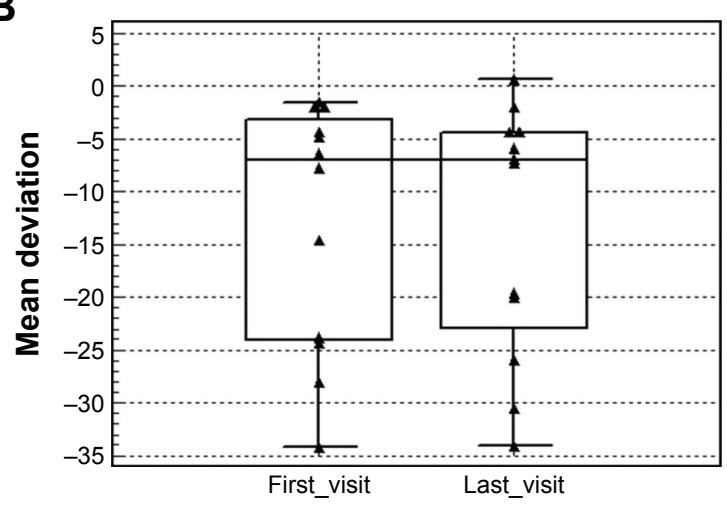

D

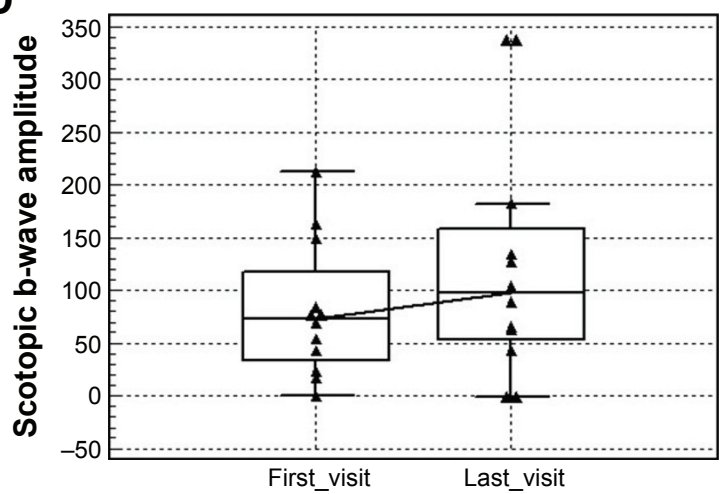

F

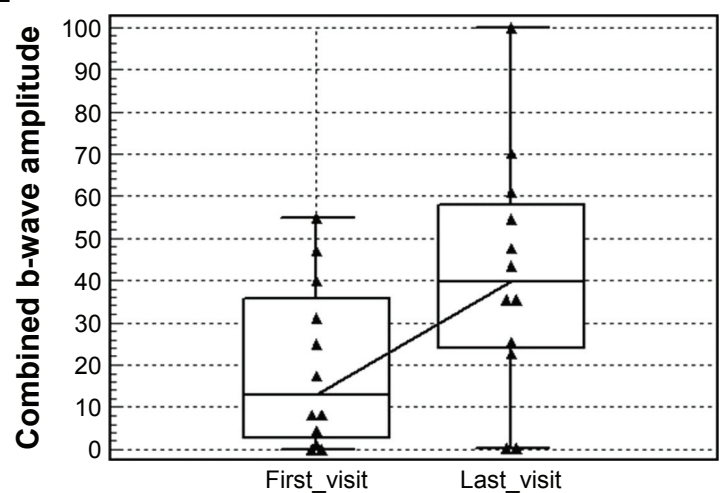

H

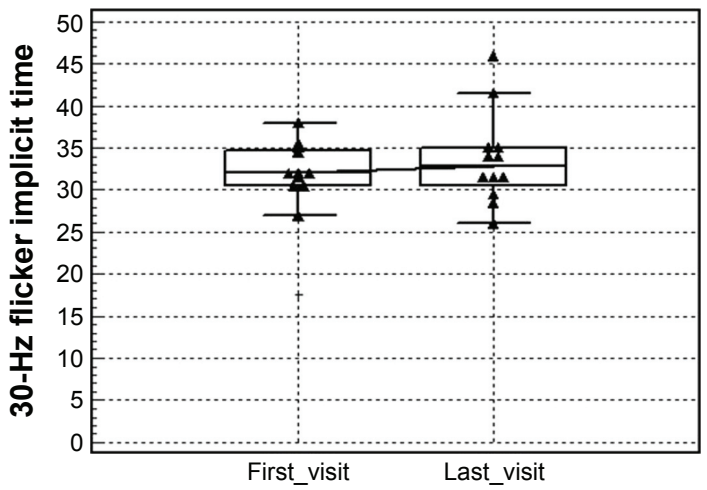

Figure I Changes in vision, visual field parameters, and electroretinography parameters from the initial visit to the most recent visit in 12 eyes of six patients with non-paraneoplastic autoimmune retinopathy. (A) Changes in LogMAR visual acuity. (B) Mean deviation changes on Humphrey visual field. (C) Pattern standard deviation changes on Humphrey visual field. (D) Changes in scotopic rod response amplitude on Ganzfeld electroretinography. (E) Changes in maximal response a-wave amplitude on Ganzfeld electroretinography. (F) Changes in maximal response b-wave amplitude on Ganzfeld electroretinography. (G) Changes in 30-Hz flicker amplitude on Ganzfeld electroretinography. (H) Changes in $30-\mathrm{Hz}$ flicker implicit time on Ganzfeld electroretinography. The central box shows the 25th-75th percentile. The middle line represents the median. Vertical line extends from the minimum to the maximum. Separate triangles show far out values (larger than the upper quartile plus three times the interquartile range). The solid line connects the median values.

Abbreviation: logMAR, logarithm of the minimum angle of resolution. 

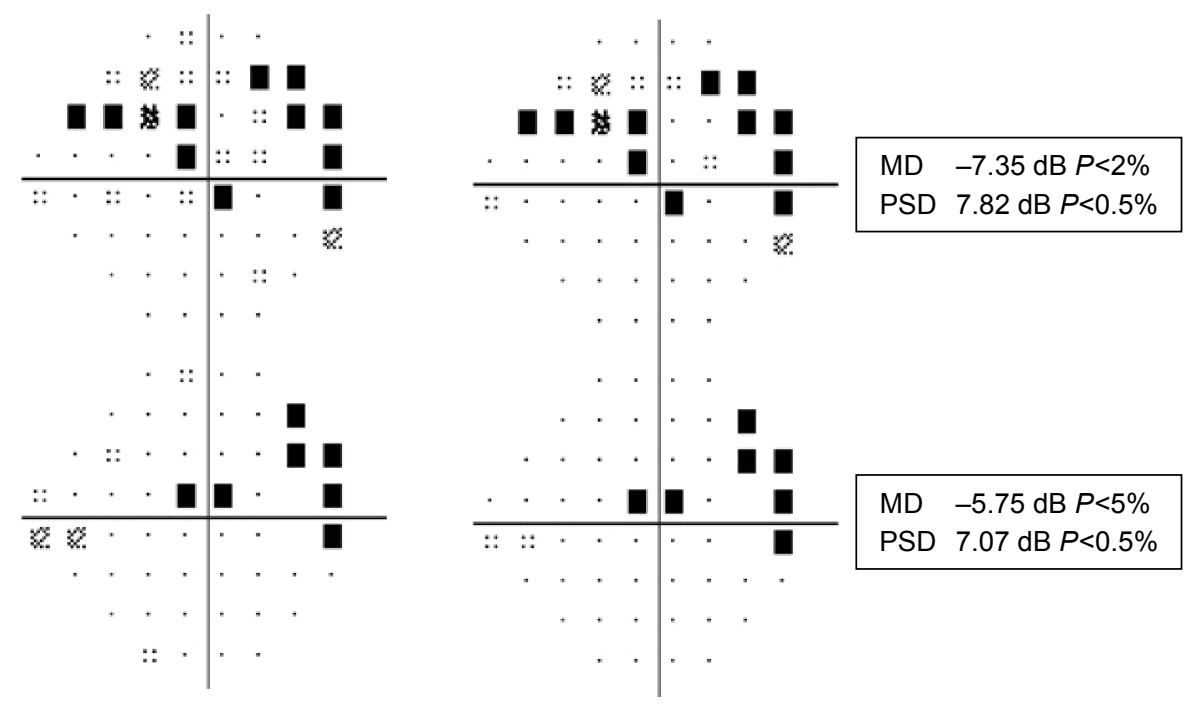

Figure 2 Changes in visual field parameters (improvement in the mean deviation and stability of pattern standard deviation) in one of the patients from the initial visit before starting rituximab (upper panel) to the last visit (lower panel).

Abbreviations: MD, mean deviation; PSD, pattern standard deviation.

showed stability/improvement (50\% of electroretinography parameters were stable and the other 50\% improved). Figure 3 shows improvement in 30-Hz flicker amplitude and implicit time on rituximab therapy based on the definition. In addition, one patient (16.7\%), who exhibited bilateral (two eyes, $16.7 \%$ ) negative waveforms on electroretinography at the initial visit, had documented improvement in one eye after treatment. One eye developed negative waveforms during the treatment. Table 3 shows changes in visual acuity, the HVF, and electroretinography as a single test modality from the initial visit to the last visit. Nine (75\%) eyes of five $(83.5 \%)$ patients showed stability or improvement in at least two test modalities, including visual acuity, visual field, and electroretinography.
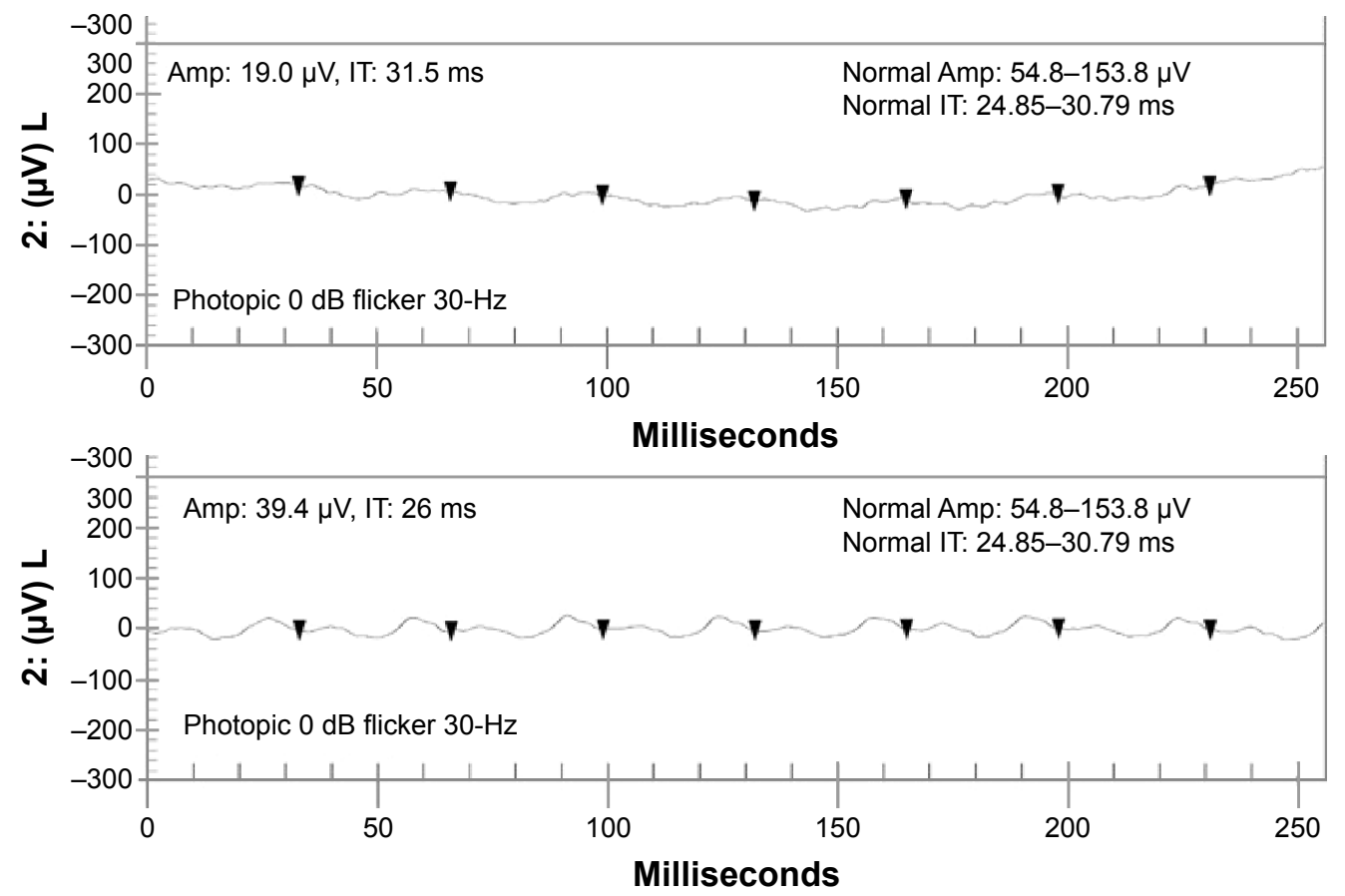

Figure 3 Changes in 30- $\mathrm{Hz}$ flicker electroretinography parameters (improvement in the Amp and IT) in one of the patients from the initial visit before starting rituximab (upper panel) to the last visit (lower panel).

Abbreviations: Amp, amplitude; IT, implicit time. 
Table 3 Changes in visual acuity, HVF and electroretinography as single tests modalities from the initial visit to the most recent visit in patients with non-paraneoplastic autoimmune retinopathy

\begin{tabular}{|c|c|c|c|}
\hline $\begin{array}{l}\text { Patient no/ } \\
\text { sex/age/eye }\end{array}$ & $\begin{array}{l}\text { Change }^{a} \\
\text { in V/A }\end{array}$ & $\begin{array}{l}\text { Change }^{a} \\
\text { in HVF }\end{array}$ & $\begin{array}{l}\text { Change }^{a} \\
\text { in ERG }\end{array}$ \\
\hline I/F/30/OD & $w$ & $\mathrm{~S}$ & $w$ \\
\hline I/F/30/OS & w & S & W \\
\hline 2/F/47/OD & $S$ & S & $\mathrm{S} / \mathrm{I}$ \\
\hline 2/F/47/OS & w & $S$ & 1 \\
\hline 3/F/5I/OD & $S$ & $W$ & $S / I$ \\
\hline 3/F/5I/OS & $S$ & $\mathrm{~S} / \mathrm{W}$ & $S$ \\
\hline 4/F/73/OD & w & S & W \\
\hline 4/F/73/OS & $S$ & S & w \\
\hline 5/M/54/OD & S & I & $S / I$ \\
\hline $5 / \mathrm{M} / 54 / \mathrm{OS}$ & S & $S / W$ & 1 \\
\hline 6/F/40/OD & S & $\mathrm{S} / \mathrm{W}$ & $S$ \\
\hline 6/F/40/OS & $S$ & I & $S / I$ \\
\hline
\end{tabular}

Note: aChange from the initial visit to the most recent visit.

Abbreviations: ERG, electroretinography; F, female; HVF, Humphrey visual field; I, improved; M, male; OD, right eye; OS, left eye; S, stable; S/I, stable/improved; S/W, stable/worse; V/A, visual acuity; W, worse.

At the initial visit just before starting rituximab, all patients were positive for anti-retinal and anti-optic nerve antibodies. The average number of anti-retinal antibody bands was $2.83 \pm 2.31$ (range, 1-7). All but one had at least one pathologically proven anti-retinal antibody band (mean, 1.5 \pm 1.04 ; range, $0-3)$. The average number of anti-optic nerve antibody bands was $2.33 \pm 1.03$ (range, $1-3$ ). The pathologically proven anti-optic nerve antibody band was found only in one patient. At the most recent visit, the average number of anti-retinal antibody bands was $2.33 \pm 1.96$ (range, $1-6$ ), and the average number of anti-optic nerve antibody bands was $1.66 \pm 1.36$ (range, 0-4). After treatment, the average number of pathologically proven anti-retinal autoantibodies bands was $1 \pm 0.89$ (range, 0-2); the average number of nonspecific anti-optic nerve antibodies was the same, although the number of bands decreased in five patients and increased in one patient. Interestingly, in three out of five patients with pathologically proven anti-retinal antibodies, at least one pathogenically proven band disappeared after treatment; however, one of these patients developed a new pathologically proven band. Table 4 describes pathologically proven and nonspecific anti-retinal and anti-optic nerve antibodies at the initial and most recent visits.

\section{Discussion}

On the basis of the presumed antibody-mediated mechanism ${ }^{2,5,6}$ and our personal experience regarding the failures of conventional immunomodulatory therapy (four patients in this cohort), we evaluated the efficacy of rituximab as a monotherapy or in combination with cyclophosphamide and/or bortezomib to preserve or improve visual functions in patients with non-paraneoplastic AIR. The rationale for this protocol was to eliminate the B cells, precursors of plasma cells that are responsible for making the autoantibodies against retinal cells. Oral cyclophosphamide and/or bortezomib were used in patients with severe disease.

Of the six patients, two patients (four eyes) were previously diagnosed with HLA-A29-positive birdshot retinochoroidopathy. These cases were in remission for a long time ( $>2$ years). Neither of them had active intraocular inflammation, especially vitreous cells and vitreous haze. They had subacute impressive visual field and visual acuity loss with normal fluorescein angiography and stable ICG angiography. These findings made us suspicious to AIR more than birdshot retinochoroidopathy relapse as AIR has previously been reported in cases with birdshot retinochoroidopathy. ${ }^{14}$ Both of these cases responded to rituximab infusions.

In our study, vision did not improve in any patient despite treatment; however, visual acuity was stable at the most recent visit in eight (66.7\%) eyes. Ferreyra et al showed improved vision in three eyes of three patients out of 15 patients with non-paraneoplastic AIR who responded to immunosuppressive therapy. ${ }^{6}$ Two patients in their study had cystoid macular edema that improved after treatment, which can explain the

Table 4 Anti-retinal and anti-optic nerve autoantibody bands in non-paraneoplastic autoimmune retinopathy

\begin{tabular}{|c|c|c|c|c|c|c|c|c|}
\hline $\begin{array}{l}\text { Patient } \\
\text { no/sex/ } \\
\text { age }\end{array}$ & $\begin{array}{l}\text { ARA bands } \\
\text { (pathologic), } \\
\text { first visit } \\
\text { (kDa) }\end{array}$ & $\begin{array}{l}\text { ARA bands } \\
\text { (pathologic), } \\
\text { last visit } \\
\text { (kDa) }\end{array}$ & $\begin{array}{l}\text { ARA bands } \\
\text { (nonspecific), } \\
\text { first visit } \\
\text { (kDa) }\end{array}$ & $\begin{array}{l}\text { ARA bands } \\
\text { (nonspecific), } \\
\text { last visit } \\
\text { (kDa) }\end{array}$ & $\begin{array}{l}\text { AOA bands } \\
\text { (pathologic), } \\
\text { first visit } \\
\text { (kDa) }\end{array}$ & $\begin{array}{l}\text { AOA bands } \\
\text { (pathologic), } \\
\text { last visit } \\
\text { (kDa) }\end{array}$ & $\begin{array}{l}\text { AOA bands } \\
\text { (nonspecific), } \\
\text { first visit } \\
\text { (kDa) }\end{array}$ & $\begin{array}{l}\text { AOA bands } \\
\text { (nonspecific), } \\
\text { last visit } \\
\text { (kDa) }\end{array}$ \\
\hline $\mathrm{I} / \mathrm{F} / 30$ & $30,46,48$ & 30,45 & $31,43,62,122$ & $43,60,62,112$ & $\mathrm{~N}$ & $N$ & $46,62,64$ & $\mathrm{~N}$ \\
\hline $2 / F / 47$ & 22 & 22 & $\mathrm{~N}$ & $\mathrm{~N}$ & 22 & 22 & $\mathrm{~N}$ & $131,132,135$ \\
\hline 3/F/5I & 40,46 & 46 & $N$ & $N$ & $N$ & $N$ & $46,50,62$ & 36,62 \\
\hline 4/F/73 & $33-35$ & $\mathrm{~N}$ & $\mathrm{~N}$ & $\mathrm{~N}$ & $\mathrm{~N}$ & $\mathrm{~N}$ & $2,104,110$ & 62 \\
\hline $5 / M / 54$ & 30,46 & 30,46 & 50,72 & 72 & $N$ & $N$ & 46,72 & 72 \\
\hline 6/F/40 & $N$ & $N$ & 62,76 & 62,76 & $\mathrm{~N}$ & $\mathrm{~N}$ & $32,96,136$ & 135 \\
\hline
\end{tabular}

Notes: 22, ARA and AOA pathologic; 30, carbonic anhydrase II; 33-35, Muller cell associated; 40, aldolase; 46, enolase; 48, arrestin.

Abbreviations: AOA, anti-optic nerve antibody; ARA, anti-retinal antibody; $F$, female; $M$, male; $N$, negative. 
improvement in visual acuity. ${ }^{6}$ However, none of our patients tested with fluorescein angiography and optical coherence tomography had cystoid macular edema. Jampol and Fishman reevaluated and reanalyzed their data in patients with nonparaneoplastic AIR without cystoid macular edema; they observed stability or improvement in $60 \%$ of studied eyes. ${ }^{24}$ In our study, four patients had previous retinal, choroidal, and retinal vasculature problems, which might explain why their vision did not improve despite treatment. Moreover, three eyes of two patients with worsening of visual acuity were in the group in which the treatment was prematurely stopped due to side effects or insurance issues.

Most of the previous studies emphasized Goldman perimetry for peripheral visual changes; however, central and paracentral scotomas have been reported in patients with AIR. ${ }^{5}$ In addition, electroretinography shows peripheral retinal function accurately; therefore, we performed 24-2 FASTPAC or SITAFAST or SITA-SWAP strategy in our patients as these tests can also indicate optic nerve involvement. Different strategies were used based on the underlying problems in our patients; for example, we obtained SITA-SWAP data in patients with birdshot retinochoroidopathy. ${ }^{15}$ This does not interfere with the interpretation of the results, as we compared the status of patients at their most recent visit with their own baseline measurements. In our study, visual field improved in two eyes of two patients. Our results are not as favorable as those found by Ferreyra et al using Goldmann perimetry; ${ }^{6}$ however, our criteria for visual field improvement were very strict. In addition, we used the HVF test, which has more parameters than Goldman perimetry, the method used by Ferreyra et al. Our findings were consistent with Jampol and Fishman reanalysis of Ferreyra et al's results, in that they found no change in Goldman perimetry with IV-4-e target in half of the patients. ${ }^{24}$ These findings make our results superior to that study when taking into account our strict criteria. Moreover, all of our patients had autoimmune-related retinopathy and optic neuropathy syndrome, which might make the prognosis poorer than that of isolated AIR.

The major advantage of this study, as opposed to previous studies on this disorder, was the comprehensive evaluation of electroretinography parameters and their changes from the initial to the final visit while considering inter-test variability. ${ }^{6,11,12,20}$ The majority of parameters were stable or improved in eight eyes $(66.7 \%)$. The four eyes with worsening of the majority of the parameters on electroretinography belonged to two patients in whom the treatment was stopped prematurely due to insurance issues or side effects. Only limited case reports and small case series reported electrophysiological data with no pathognomonic findings; in addition, most of our patients were asymptomatic in the most recent visit. As such, the importance of this finding is not completely clear. The largest cohort of non-paraneoplastic AIR patients, performed by Ferreyra et al, did not include electroretinography parameters in the improvement criteria in all of their patients. ${ }^{6}$

We divided anti-retinal and anti-optic nerve antibodies into pathologically proven and nonspecific groups based on the previous studies. ${ }^{2,5,14}$ The importance of nonspecific anti-retinal and anti-optic nerve antibodies is unclear. Half of our patients showed elimination of at least one pathologically proven anti-retinal antibody band (Table 4). This can indicate the efficacy of treatment; however, due to the rarity of the disease, lack of standardization of these tests, and low antibody-specific concordance rate, this is still an area of debate. ${ }^{8,25}$ Although antibody titers may be more valuable than the bands themselves in reporting efficacy of treatment, titers were not available in antibody reports from the laboratory.

Despite all of these controversies, stability or improvement of two or more tests in nine (75\%) eyes of five $(83.5 \%)$ patients along with the reduction of pathologically proven antibody bands $(50 \%)$ can be considered a success in the treatment of this disease, which historically has had a poor prognosis. This becomes more significant if we consider that all three eyes with worsening of at least two test modalities were in the group in which the treatment was prematurely stopped. However, due to ongoing underlying autoimmune processes and the unpredictability of this rare disorder, the duration of treatment and the final prognoses of these patients are still unclear.

Our study was limited by its retrospective nature and small sample size. Not having optical coherence tomography data for all patients was another limitation of our study. We were also dependent on a commercially available laboratory for anti-retina and anti-optic nerve antibodies, and they did not include the antibodies' titers in their reports. Moreover, the patient population in our tertiary referral center is skewed toward patients with very severe intraocular disease. Thus, it is difficult to predict from our data, the outcomes of treatment in patients with a milder disease course. Notwithstanding these limitations, this article describes the only cohort to date of patients with non-paraneoplastic AIR who were treated with rituximab as a monotherapy or in combination with cyclophosphamide and/or bortezomib with a comprehensive analysis of the static visual field, electroretinography, and anti-retinal and anti-optic nerve antibodies.

Overall, stability of vision, visual field, and electroretinography parameters were observed in a high number of eyes (75\%) who were treated with rituximab as monotherapy (one 
patient) or combination therapy (four patients). However, a prospective multi-center study with a large population and longer follow-up would help us to standardize the use of visual field, electroretinography parameters, and evaluation of anti-retinal and anti-optic nerve antibody bands and titers so that criteria can be established for a proper response to treatment with rituximab, alone or in combination therapy.

\section{Disclosure}

C Stephen Foster discloses the following: consultancies with Aldeyra Therapeutics (Lexington, MA, USA), Bausch \& Lomb Surgical, Inc (Rancho Cucamonga, CA, USA), Eyegate Pharma (Waltham, MA, USA), Novartis Pharmaceuticals (Cambridge, MA, USA), pSivida (Watertown, MA, USA), and Xoma (Berkeley, CA, USA).

Grants or grants pending with Alcon (Aliso Viejo, CA, USA), Aldeyra Therapeutics, Bausch \& Lomb (Bridgewater, NJ, USA), Clearside Biomedical (Alpharetta, GA, USA), Dompé pharmaceutical (Milan, Italy), Eyegate Pharma, Icon (Dublin, Ireland), Novartis Pharmaceuticals, pSivida, Santen (Osaka, Japan), and Xoma.

Payment for lectures including service on speaking bureaus: Alcon, Allergan (Dublin, Ireland). Stock or Stock Options: Eyegate Pharma. The other authors report no conflicts of interest in this work.

\section{References}

1. Weleber RG, Watzke RC, Shults WT, et al. Clinical and electrophysiologic characterization of paraneoplastic and autoimmune retinopathies associated with antienolase antibodies. Am J Ophthalmol. 2005; 139(5):780-794.

2. Heckenlively JR, Ferreyra HA. Autoimmune retinopathy: a review and summary. Semin Immunopathol. 2008;30(2):127-134.

3. Adamus G. Autoantibody-induced apoptosis as a possible mechanism of autoimmune retinopathy. Autoimmun Rev. 2003;2(2):63-68.

4. Adamus G, Ren G, Weleber RG. Autoantibodies against retinal proteins in paraneoplastic and autoimmune retinopathy. BMC Ophthalmol. 2004; 4:1-9.

5. Grange L, Dalal M, Nussenblatt RB, Sen HN. Autoimmune retinopathy. Am J Ophthalmol. 2014;157(2):266-272.

6. Ferreyra HA, Jayasundera T, Khan NW, et al. Management of autoimmune retinopathies with immunosuppression. Arch Ophthalmol. 2009;127(4):390-397.

7. Rahimy E, Sarraf D. Paraneoplastic and non-paraneoplastic retinopathy and optic neuropathy: evaluation and management. Surv Ophthalmol. 2013;58(5):430-458.

Clinical Ophthalmology

\section{Publish your work in this journal}

Clinical Ophthalmology is an international, peer-reviewed journa covering all subspecialties within ophthalmology. Key topics include: Optometry; Visual science; Pharmacology and drug therapy in eye diseases; Basic Sciences; Primary and Secondary eye care; Patient Safety and Quality of Care Improvements. This journal is indexed on Submit your manuscript here: http://www.dovepress.com/clinical-ophthalmology-journal
8. Sawyer RA, Selhorst JB, Zimmerman LE, Hoyt WF. Blindness caused by photoreceptor degeneration as a remote effect of cancer. $\mathrm{Am} J$ Ophthalmol. 1976;81(5):606-613.

9. Murphy MA, Thirkill CE, Hart WM Jr. Paraneoplastic retinopathy: a novel autoantibody reaction associated with small-cell lung carcinoma. J Neurophthalmol. 1997;17(2):77-83.

10. Guy J, Aptsiauri N. Treatment of paraneoplastic visual loss with intravenous immunoglobulin: report of 3 cases. Arch Ophthalmol. 1999;117(4): 471-477.

11. Dy I, Chintapatla R, Preeshagul I, Becker D. Treatment of cancerassociated retinopathy with rituximab. J Natl Compr Canc Netw. 2013; 11(11):1320-1324.

12. Or C, Collins DR, Merkur AB, et al. Intravenous rituximab for the treatment of cancer-associated retinopathy. Can J Ophthalmol. 2013; 48(2):e35-e38.

13. Fox A, Jeffrey B, Hasni S, Nussenblatt R, Sen HN. Rituximab treatment for nonparaneoplastic autoimmune retinopathy. Can J Ophthalmol. 2015;50(6):e101-e104.

14. Grewal DS, Fishman GA, Jampol LM. Autoimmune retinopathy and antiretinal antibodies: a review. Retina. 2014;34(5):827-845.

15. Maleki A, Swan RT, Silpa-Archa S, et al. Short-wavelength automated perimetry parameters at baseline and following remission in patients with birdshot retinochoroidopathy. Am J Ophthalmol. 2016;163: 83.e6-92.e6.

16. Keltner JL, Johnson CA, Beck RW, Cleary PA, Spurr JO. Quality control functions of the visual field reading center (VFRC) for the Optic Neuritis Treatment Trial (ONTT). Control Clin Trials. 1993;14(2):143-159.

17. Keltner JL, Johnson CA, Spurr JO, Beck RW; Optic Neuritis Study Group. Baseline visual field profile of optic neuritis. The experience of the optic neuritis treatment trial. Arch Ophthalmol. 1993;111(2): 231-234.

18. Alencar LM, Medeiros FA. The role of standard automated perimetry and newer functional methods for glaucoma diagnosis and follow-up. Indian J Ophthalmol. 2011;(Suppl 59):S53-S58.

19. Liu S, Lam S, Weinreb RN, et al. Comparison of standard automated perimetry, frequency-doubling technology perimetry, and shortwavelength automated perimetry for detection of glaucoma. Invest Ophthalmol Vis Sci. 2011;52(10):7325-7331.

20. Foster CS, Chang PY, Ahmed AR. Combination of rituximab and intravenous immunoglobulin for recalcitrant ocular cicatricial pemphigoid: a preliminary report. Ophthalmology. 2010;117(5):861-869.

21. Cao JH, Oray M, Cocho L, Foster CS. Rituximab in the treatment of refractory noninfectious scleritis. Am J Ophthalmol. 2016;164:22-28.

22. Mills RP, Budenz DL, Lee PP, et al. Categorizing the stage of glaucoma from pre-diagnosis to end-stage disease. Am J Ophthalmol. 2006;141(1): $24-30$.

23. Fishman GA, Chappelow AV, Anderson RJ, Rotenstreich Y, Derlacki DJ. Short-term inter-visit variability of erg amplitudes in normal subjects and patients with retinitis pigmentosa. Retina. 2005; 25(8):1014-1021.

24. Jampol LM, Fishman GA. Immunosuppression for autoimmune retinopathy. Arch Ophthalmol. 2009;127(4):573-575.

25. Faez S, Loewenstein J, Sobrin L. Concordance of antiretinal antibody testing results between laboratories in autoimmune retinoapathy. JAMA Ophthalmol. 2013;131(1):113-115. 